\title{
An Analysis of the Elastic Medium Dispersion Phenomenon in the Cased Well
}

\author{
Danyan Xie ${ }^{1, a, *}$, Wei Yang ${ }^{2, b}$ and Jin Qian ${ }^{1, c}$ \\ ${ }^{1}$ College of Computer Science and Technology, Taizhou University, Hailing District, Taizhou City, China \\ ${ }^{2}$ Nanjing Yunduopu Network Co., Ltd., Jianye District ,Nanjing City, China

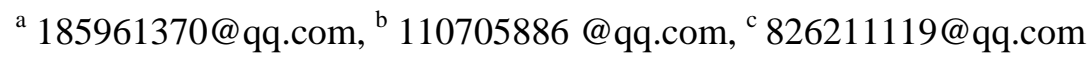

Keywords: Cut-off frequency, Stanley wave, Pseudo Rayleigh wave, Dispersion curve.

\begin{abstract}
The Real axis integral method is used to simulate the frequency dispersion curve of the axial symmetry cased well, analyze the frequency dispersion characteristics of the axial symmetry wellhole model with different channel categories. It has been found that both stoneley wave and pseudo-Rayleigh wave have the cut-off frequency. When the wellhole has channel, a large amount of low-speed stoneley wave exists under the stoneley wave, and splashes indicating various channel characteristics happen in the low-frequency scope. Through comparing the data of the scale well and actual measurement well, it can be proved that the frequency dispersion phenomenon can also be conducted with well cementation quality evaluation, which is more visualized than the full-wave evaluation.
\end{abstract}

\section{Introduction}

Acoustic logging is a very important well measuring method. It is widely used in exploring,mining the oil field, engineering geophysical exploration and many other fields. Acoustic logging utilizes the acoustic parameters, such as the amplitude attenuation, speed or frequency change when the acoustic wave is communicating in the casing pipe, cement sheath, rock and numerous other mediums to study the geological section of the well, judge well cementation quality and other engineering problems. In order to obtain the correct information of wellhole and stratum from the well logging signal, it is required to have in-depth understanding to the communication regulation of the acoustic wave in wellhole, so as to grasp the influence of formation lithology and wellhole property to acoustic wave communication.[1,2]

Wellhole acoustics is the theoretical basis of acoustic logging. The geometric construction of the wellhole and the property of the geological medium out of the hole decide the communication mechanism of the acoustic wave in wellhole. For decades, many domestic and foreign scholars have conducted a large amount of theoretical analysis, numerical calculation and experimental work in this aspect. $[3,4]$

Real axis integral method is used to simulate the wellhole sound field, two-dimension spectrum and frequency diversion curve of the axial symmetry cased well, analyze the frequency dispersion characteristics of the axial symmetry wellhole model with different channel categories[5,6,7]. It has been found that both stoneley wave and pseudo-Rayleigh wave have the cut-off frequency. When the wellhole has channel, a large amount of low-speed stoneley wave exists under the stoneley wave, and splashes indicating various channel characteristics happen in the low-frequency scope.

\section{Wellbore model and media parameters}

The cased hole model is as shown in Fig. 1 . The radius of the well is $0.06285 \mathrm{~m}$, the thickness of the steel casing is $0.007 \mathrm{~m}$, and the thickness of the cement layer is $0.03 \mathrm{~m}$. The medium from the inside to the outside in turn represent the borehole fluid, steel casing, cement ring, formation. The sound source adopts Gaussian envelope, the center frequency is $18 \mathrm{KHz}$, and the bandwidth is $6 \mathrm{KHz}$. The media parameters are shown in Table 1. 


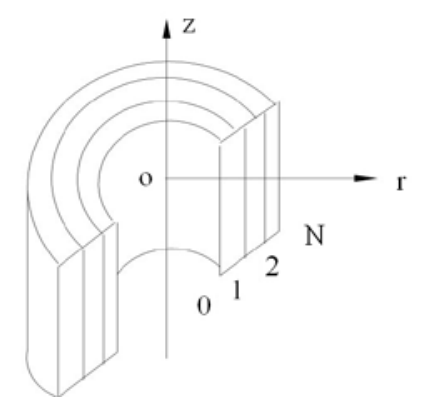

Figure 1 Cased hole model.

Table.1 Medium parameters

\begin{tabular}{|c|c|c|c|c|c|}
\hline media & $\begin{array}{c}\text { P - wave } \\
\text { velocity (m/s) }\end{array}$ & $\begin{array}{c}\text { S - wave } \\
\text { velocity (m/s) }\end{array}$ & $\begin{array}{c}\text { density } \\
(\mathrm{kg} / \mathrm{m} 3)\end{array}$ & Qp & Qs \\
\hline well fluid & 1580 & 30 & 1090 & 50 & 50 \\
\hline steel casing & 6098 & 3354 & 7500 & 200 & 200 \\
\hline cement layer & 2800 & 1700 & 1920 & 80 & 80 \\
\hline fast formation & 3800 & 2200 & 2600 & 30 & 30 \\
\hline
\end{tabular}

\section{Real axis integration method and dispersion equation}

\section{1. Real axis integration method[8]}

The sound wave equation of the fluid in the well as shown in Eq. 1.

$$
\nabla^{2} p=\frac{\partial^{2} p}{v_{f}^{2} \partial t^{2}}
$$

$P$ is the sound pressure. It is formed by the direct wave sound pressure $p_{o}$ and the reflected wave sound pressure $p_{f}$. If the external medium is a solid elastic medium, the elastic wave field of the nth layer solid elastic medium as shown in Eq. 2.

$$
\left(\lambda_{n}+2 \mu_{n}\right) \nabla\left(\nabla \times \nabla \cdot u_{n}\right)-\mu_{n} \nabla \times \nabla \times u_{n}-\rho_{n} \frac{\partial^{2} u_{n}}{\partial t^{2}}=0
$$

$\lambda_{n}$ and $\mu_{n}$ represent the pull density factor of the nth layer solid elastic medium.

Calculate the P-wave and S-wave displacements of the sound field, that is, two two-dimensional spectra of the sound field outside the well. Then through the double Fourier inverse transform, the displacement of the fluid in the well as shown in Eq. 3.

$$
p_{0}(r, z, t)=\frac{1}{2 \pi} \int_{-\infty}^{+\infty} \int_{-\infty}^{+\infty} \frac{i}{2 \rho_{0} \omega^{2}} F(\omega)\left[H_{0}^{(1)}\left(\alpha_{0} r\right)+A_{0}(k, \omega) J_{0}\left(\alpha_{0} r\right)\right] \exp (i k z-i \omega t) d k d \omega
$$

Sound pressure in the well as shown in Eq. 4.

$$
p(r, z, t)=\frac{1}{2 \pi} \int_{-\infty}^{+\infty} \int_{-\infty}^{+\infty} F(\omega)\left[H_{0}^{(1)}\left(\alpha_{0} r\right)+A_{0}(k, \omega) J_{0}\left(\alpha_{0} r\right)\right] \exp (i k z-i \omega t) d k d \omega
$$

Calculate the sound field of each layer outside the well by boundary conditions and transfer matrix.

\subsection{Dispersion equation}

When the M matrix in the real axis integral derivation is 0, as shown in Eq.5.

$$
M\left[\begin{array}{l}
A \\
B \\
i k C
\end{array}\right]=0,|M|=0
$$


This is an equation about the angular frequency $\omega=2 \pi f$ and the wave number $k, f$ is the frequency. That is dispersion equation. A non-zero eigenvector composed of $\mathrm{A}, \mathrm{B}$, and $\mathrm{C}$ can determine the sound field of the pattern, $k, f$ are the frequency and wave number.

Solving the dispersion equation, we get the relationship between the frequency wave number or the frequency velocity, which is the dispersion curve. Therefore, the dispersion curve is generally a relationship of wave number-frequency or velocity -frequency.[9,10]

\section{Analysis of the sound field and frequency dispersion curve of the cased well}
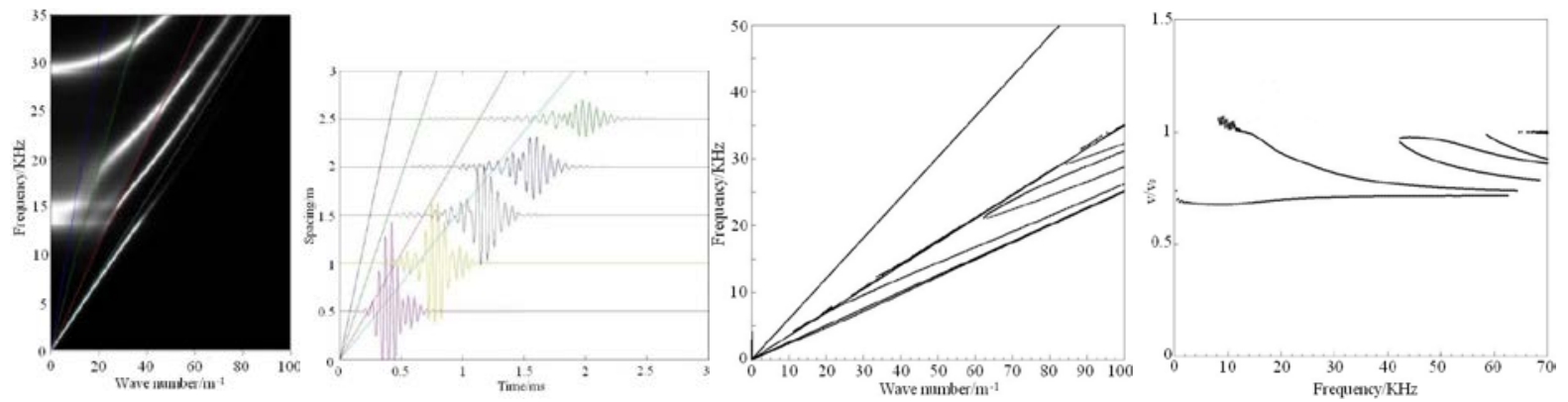

Figure 2 Sound field of well cementing (a) Two-dimensional spectrum (b) Waveforms (c) Dispersion curves (d) The phase velocity of propagation mode.

\subsection{Sound field and frequency dispersion curve of the well cemented cased wellhole}

The sound field of the well cemented cased well is as shown in Fig. 2. (a) Two-dimensional spectrum (b) Waveforms (c) Dispersion curves (d) The phase velocity of propagation mode, in which, the later two are called as the frequency dispersion curves.

Fig. 2(a) is the two-dimension spectrum on the well shaft of the cemented cased well. The slopes of the four skew lines respectively represent the P-wave of the steel sleeve, P-wave and S-wave of the stratum, as well as the P-wave speed of the fluid in the hole from the left to the right. We can clearly see that: All modes have one cut-off frequency. A closer distance to the cut-off frequency leads to a weaker communication signal of the mode. The phase speeds of all modes are not lower than the phase speed of the slurry wave. The zero-order mode is at the bottom, which is almost a straight line from $0 \mathrm{~Hz}$. The first-order mode starts from $8-10 \mathrm{KHz}$. The second-order mode starts from $16-18 \mathrm{KHz}$. The third-order mode starts from $30 \mathrm{KHz}$. From the wellhole mode with rigid wall, it can be concluded that, the zero-order communication mode in the wellhole is in a straight-line format. Other modes should originally have perfect arc. However, the first-order mode has bifurcation. If the reason is analyzed, it must be caused by the coupling deformation of its communication mode(mainly S-wave of the stratum) in the medium out of the well. Fig. 2(b) is the full acoustic waveform of the wellhole center axis with different spaces. It is visible that every full acoustic wave is roughly divided into three parts. The first part has rather weak signal. Its speed is approaching the P-wave of the solid elastic medium out of the well. The second part has rather strong signal. Its speed is approaching the S-wave of the solid elastic medium out of the well. The third part has the strongest signal. Its speed is approaching the P-wave speed of the fluid in the well. When the wellhole is well cemented, the signal communicating along the steel sleeve and cement layer is not significant. However, the signal of stratum wave is very clear. The signal strength of the stratum P-wave is slightly weaker than the signal strength of the stratum S-wave. There is a very strong signal in the tail, which is considered to be the slurry wave communicating along the fluid in the well.

Fig. 2(c) and Fig. 2(d) are respectively the wave number-frequency domain frequency dispersion curve and the phase speed. From Fig. 2(d), we can find that the lowest stoneley wave exists in the frequency scope of $0-60 \mathrm{KHz}$, and aborts when the frequency exceeds $60 \mathrm{KHz}$. In this scope, the phase speed has very small change, namely, the frequency dispersion is very weak. When the frequency is $0 \mathrm{~Hz}$, the phase speed is slightly lower than the speed of P-wave of the in-hole fluid $v_{f}$, 
which is about to $0.6 v_{s}$. As the frequency increases gradually, it tends to approach the $v_{f}$. The lines above stoneley wave are pseudo-Rayleigh waves. Each pseudo-Rayleigh wave has the cut-off frequency. The phase speed of the cut-off frequency is approaching to the S-wave of the stratum $v_{s}$. As the frequency increases, pseudo-Rayleigh wave decreases slowly and eventually approaches to the sound speed of the fluid $v_{f}$.

\subsection{Frequency dispersion curve of the I interface channel}

Channeling on the I interface means that there is a thin water layer between the steel sleeve and cement layer. Just assume that the water layer has the thickness of $0.01 \mathrm{~m}$, the value of the medium parameter is the same as the fluid in the well, the thickness of the cement layer is reduced to $0.01 \mathrm{~m}$ accordingly. Then the thickness of the cement layer is $0.02 \mathrm{~m}$. Then the frequency dispersion curve of the I interface channel of the cased well can be calculated, as shown in Fig. 3.
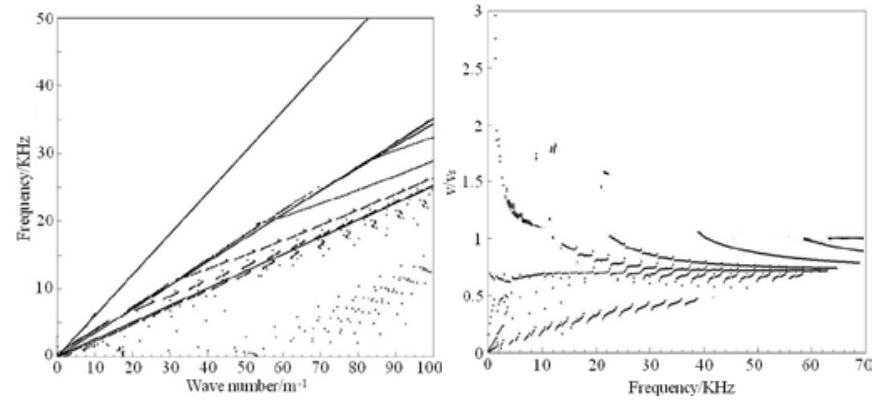

Figure 3 The I interface channel (a) Dispersion curves (b) The phase velocity of propagation mode.

Fig. 3(a) and Fig. 3(b) are respectively the wave number-frequency domain frequency dispersion curve of the I interface channel wellhole and the phase speed of wellhole. On the basis of well cementation, the frequency dispersion curve of I interface channel still has corresponding changes: When I interface has channel, in addition to the stoneley wave and several pseudo-Rayleigh waves, there are several other communication modes in the figure. These communication modes are in the scope of lower than the stoneley wave. We also regard them as the stoneley waves. Moreover, in case of channel, some isolated splashes still exist in the figure of the phase speed. For example, in Fig.3(b), in the scope of the frequency approaching 1.5-10KHz, there are still some splashes with $v / v_{s}$ higher than 1 , in which, there are three points of $v / v_{s} \approx 2.6, v / v_{s} \approx 2.75, v / v_{s} \approx 2.9$, namely, $v \approx 5700 \mathrm{~m} / \mathrm{s}, \quad v \approx 6050 \mathrm{~m} / \mathrm{s}, \quad v \approx 6380 \mathrm{~m} / \mathrm{s}$. We believe that this is the communication mode of the casing pipe wave. As the frequency increases, the speed of these splashes also reduces gradually. In addition to these three points, we still have some points of $v / v_{s} \approx 2.0, v / v_{s} \approx 1.6$, namely, points of $v \approx 4040 \mathrm{~m} / \mathrm{s}, \quad v \approx 3520 \mathrm{~m} / \mathrm{s}$. We believe that these points are the communication mode of the P-wave of the stratum.

\subsection{Frequency dispersion curve of II interface channel of the cased well.}

Channeling on the II interface means that there is a thin water layer between the cement layer and stratum. The frequency dispersion curve of the II interface channel of the cased well is obtained through calculation, just as shown in Fig. 4.
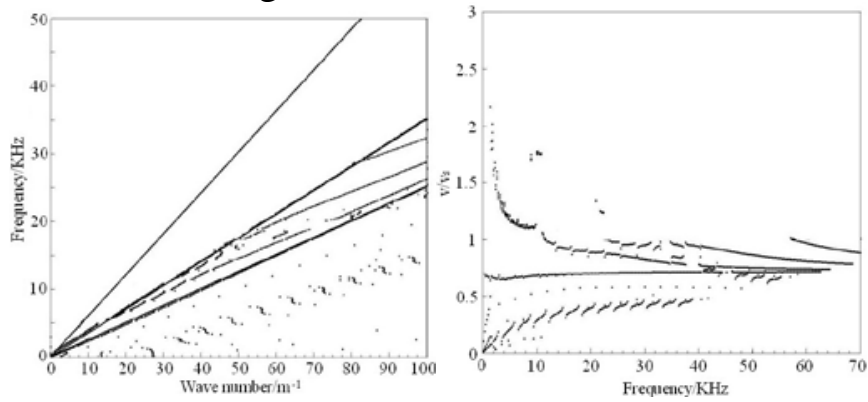

Figure 4 The II interface channel (a) Dispersion curves (b) The phase velocity of propagation mode. 
Fig.4(a) and Fig.4(b) are respectively the wave number-frequency domain frequency dispersion curve and the phase speed. When II interface has channel, in addition to the stoneley wave and several pseudo-Rayleigh waves, there are several other communication modes, which is similar to the frequency dispersion curve of the I interface channel. These communication modes are in the scope of lower than the stoneley wave. We also regard them as the stoneley waves. Moreover, in case of channel, some isolated splashes still exist in the figure of the phase speed. In Fig.4 (b), when the frequency approaches to the scope of $1.5-10 \mathrm{KHz}$, there are also some splashes. Its $v / v_{s} \approx 2.2$, namely, $v \approx 4840 \mathrm{~m} / \mathrm{s}$, which is similar to the model wave between the casing pipe and cement sheath. From the figure, it is visible that the cement wave of the casing pipe is dispersed. The communication of the P-wave of the stratum also exists in the lower position of this model wave.

\subsection{Frequency conversion curve with channel on I and II interfaces of the cased well}

Fig. 5 is the frequency conversion curve with channel on both I and II interfaces of the cased well. When the two interfaces have channel, it means that the splashes of the casing pipe wave of I interface channel and the characteristic wave of II interface channel exist.
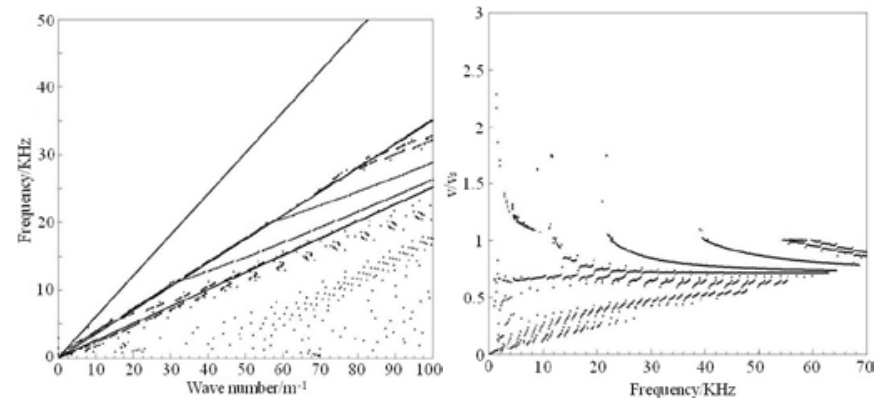

Figure 5 The I II interface channel (a) Dispersion curves (b) The phase velocity of propagation mode.

\section{Result of scale well experiment and measured data in the field}

\subsection{Contrast between the numerical simulation result and scale well experiment and measured data}

in order to illustrate the reliability of the numerical simulation result, it is required to use the experiment result and measured data in the field for verification. The experiment done in this paper is conducted in the scale well group in Daqing Oil Field. The scale well group includes 1\# 4\# scale well, in which, $1 \#$ is in the full cemented status of the formation lithology of $139.7 \mathrm{~mm}$ with different casing pipes(include sandstone, mudstone and limestone), $2 \#$ and $3 \#$ are in the status of having angle cementation deficiency on the I interface of the casing pipe mudstone stratum in $139.7 \mathrm{~mm}, 4 \#$ is that the casing pipe mudstone stratum has cement angle cementation loss condition on the II interface. In the experiment, DLS-III acoustic wave density log instrument is used to measure 1\#, 2\#, 3\# and 4\# wells. Emphasis has been given to analyze the conditions of well cementation, channel on I interface, channel on II interface and so on. As shown in Fig. 6(a). In addition, these measured data is compared with the numerical simulation result. Fig.6(b) displays the result contrast between the scale well and numerical simulation result. 

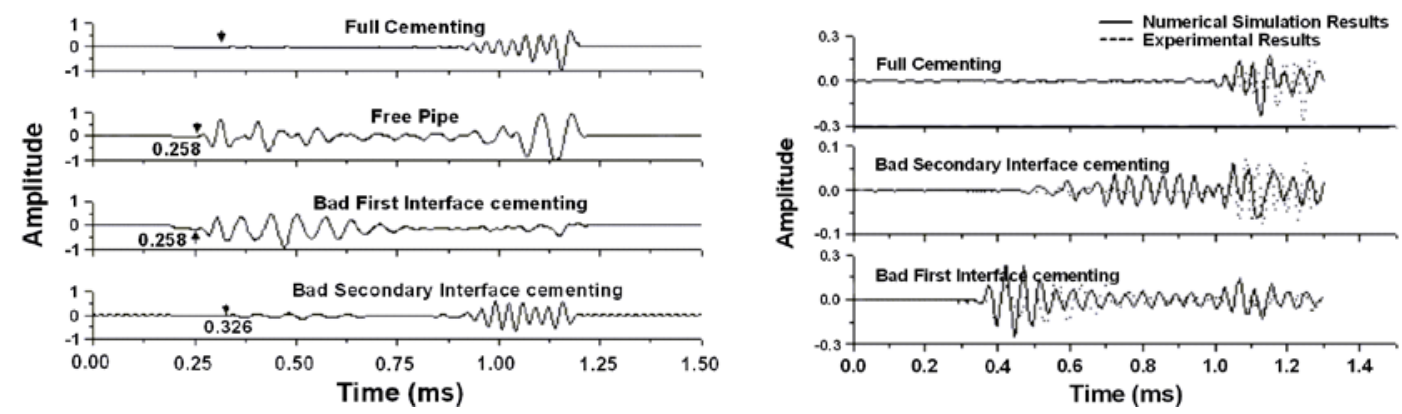

Figure 6 (a)The experimental results of Scale well (b)Contradistinction of the numerical simulation results and the experimental results of Scale well.

\subsection{Analysis of the measured acoustic full wave}

Through numerical simulation and measured data waveform analysis of the scale well, a preliminary conclusion can be reached: The cementation quality of the I and II interfaces is displayed in the frequency dispersion curve. However, such a viewpoint shall be inspected by the local measured materials. For this purpose, we have analyzed the actual data in the field, and seen the waveform characteristics of the II interface channel being reflected before in some acoustic change logging materials of the cased well. For instance, the acoustic change time-lapse logging of the south 3 water 453 well of Daqing Oil Field is shown in Fig.7. The waveforms in Fig.7 are indeed three logging results of the same well. The logging time is indeed the 2th, 7th and 15th days after the cementation. In the waveform measured in the 7th day, the preliminary wave of $877-883 \mathrm{~m}$ well section is the cased well, showing that I interface has channel. The arrival time of the preliminary wave below $883 \mathrm{~m}$ is later than that of the upper well section, but is significantly earlier than the stratum wave. It can be inferred that this is caused by II interface channel. The waveform measured in the 15th day also has the same characteristics. The waveform changing characteristics are basically consistent with the numerical simulation and scale well test data.

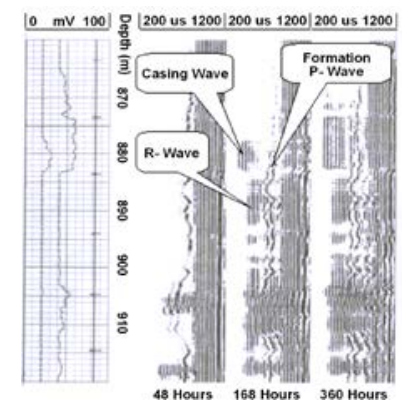

Figure 7 VDL waveform comparison of variable density time log.

\section{Conclusion}

The real axis integral method is used to simulate the frequency disperse curves in the circumstances of well cementation of the cased well, as well as various channel. The results show that: First, in the cased well with well cementation, the frequency scope of stoneley wave is approximately $0-60 \mathrm{KHz}$. And it aborts when the frequency exceeds $60 \mathrm{KHz}$. When the frequency is $0 \mathrm{~Hz}$, the phase speed is slightly lower than the P-wave speed of the in-hole fluid, which is equivalent to $0.6 v_{s}$ the stratum. As the frequency slowly increases, it eventually approaches the Pwave speed. Second, The pseudo-Rayleigh wave is above the stoneley wave. Every pseudoRayleigh wave has a cut-off frequency. The phase speed of the cut-off frequency approaches to the transverse speed of the stratum. The phase speed of pseudo-Rayleigh wave gradually decreases as the frequency increases, and eventually approaches to the P-wave of the fluid. Third, when the cased well has channel, there will be a large amount of low-speed stoneley waves below the stoneley wave. There are still some splashes with the phase speed of higher than 1 after normalization, representing the casing pipe wave of I interface channel, characteristic wave of II 
interface channel and so on. The result basically coincides with full wave analysis result.

In order to validate the accuracy of the simulation result, the numerical simulation result is compared with the test result of the scale well and measured data in the field. It has been proved that the cementation quality of I and II interfaces of the well cementation have been displayed in the frequency dispersion curve. The frequency dispersion curve can also assess the quality of well cementation, with a more visualized result than the analysis of the full wave.

\section{Acknowledgements}

This work is supported by NSFC (Grant Nos. 61802274), Natural Science Research Project of Jiangsu Province of China (Grant No. 18KJB510044).

\section{References}

[1] Bryant, H.L. (1960) Production Well Logging Techniques. Geophysics, 25, 905-927.

[2] Frisch, G.J., Graham, W.L., Griffith, J. ( 1999) Assessment of Foamed-cement Slurries Using Conventional Cement Evaluation Logs and Improved Interpretation Methods. SPE55649 Presented at the SPE Rocky Mountain Regional Meeting, Wyoming, America,509-518.

[3] Tubman, K.M., Cheng, C.H., Cole, S.P. (1986) Synthetic Full-waveform Acoustic Logs in Cased Boreholes, II-Poorly Bonded Casing. Geophysics, 51, 902-913.

[4] Lin, W.J., Zhang, C.Y., Zhang, H.N. (2005) Acoustic Field in A Cased Well with A Sectorial Crossing Channel. Acta Acoustic ,30, 9-14.

[5] Lin, W.J., Wang, X.M., Zhang, H.N. (2006)Acoustic Wave Propagation in A Borehole Penetrating An Inclined Layered Formation. Chinese Journal of Geophysics ,49,284-294.

[6] Zhang, H.L., Wang, X.M., Zhang, B.X. (2004) Acoustic Field and Waveform in the Borehole. Science Press in Beijing.

[7] Ekstrom, M.P. (1995) Dispersion Estimation from Borehole Acoustic Arrays Using A Modified Matrix Pencil Algorithm. Proceedings of the 29th Asilomar Conference on Signals. Systems and Computer Washington DC,USA. 499-453.

[8] Zhang,B.X., Xiao,B.X., Yang,W.J., Cao,S.Y., Mou,Y.G. (2000) Mechanism of Zigzag Dispersion Curves in Rayleigh Exploration and Its Inversion Study. Chinese Journal of geophysics,43(4), 557-567.

[9] Liu, L., Lin,W.J., Zhang,H.L., Wang,X.M. (2015) Mode Analyses in A Deviated Borehole Penetrating A Transversely Isotropic. Progress in Geophysics, 30(2),797-804.

[10] Zhang, B.X.,Wang, K.X. (2000) Theoretical Study of Multiple Acoustic Logging in Anisotropic Formation. Progress in Geophysics,13(2),1-14. 